ISSN 0103-5150

Fisioter. Mov., Curitiba, v. 25, n. 3, p. 679-688, jul./set. 2012 Licenciado sob uma Licença Creative Commons

\title{
Relação entre hiperpronação subtalar e lesões do ligamento cruzado anterior do joelho: revisão de literatura
}

\author{
Relationship between subtalar hyperpronation and injuries on \\ the anterior cruciate ligament of the knee: literature review
}

\section{Rodrigo Scattone da Silva ${ }^{[a]}$, Ana Luisa Granado Ferreira ${ }^{[b]}$, Lívia Maria Veronese ${ }^{[c]}$, Patrícia Driusso $^{[\mathrm{d}]}$, Fábio Viadanna Serrão ${ }^{[\mathrm{e}]}$}

[a] Fisioterapeuta, mestre em Fisioterapia pela Universidade Federal de São Carlos (UFSCar), São Carlos, SP - Brasil, e-mail: scattone@fcm.unicamp.br

[b] Graduanda em Fisioterapia pela Universidade Federal de São Carlos (UFSCar), São Carlos, SP - Brasil, e-mail: anagranado@live.com

[c] Graduanda em Fisioterapia pela Universidade Federal de São Carlos (UFSCar), São Carlos, SP - Brasil, e-mail: livia_veronese@hotmail.com

[d] Fisioterapeuta, doutora em Ciências Fisiológicas pela Universidade Federal de São Carlos (UFSCar), professora adjunta do Departamento de Fisioterapia da UFSCar, São Carlos, SP - Brasil, e-mail: pdriusso@ufscar.br

[e] Fisioterapeuta, doutor em Fisioterapia pela Universidade Federal de São Carlos (UFSCar), professor adjunto do Departamento de Fisioterapia da UFSCar, São Carlos, SP - Brasil, e-mail: fserrao@ufscar.br

\section{Resumo}

Introdução: A ruptura do ligamento cruzado anterior (LCA) é uma lesão severa, que resulta em instabilidade funcional e distúrbios articulares degenerativos. Fatores de risco proximais à articulação do joelho têm sido bastante enfatizados na última década, mas pouca atenção tem sido dada para os fatores de risco distais ao joelho. A hiperpronação subtalar (HS) foi sugerida por alguns autores como possível fator de risco às lesões do LCA, mas as evidências da literatura a respeito são escassas e pouco conclusivas. Objetivo: O propósito deste estudo foi realizar uma revisão dos estudos da literatura que avaliaram as alterações de alinhamento da articulação subtalar associadas à lesão do LCA ou associadas a outros fatores de risco 
conhecidos para essa lesão. Materiais e métodos: Foi realizada uma busca em bases eletrônicas (PubMed, MEDLINE, COCHRANE, Web of Science, PEDro, SciELO, LILACS e EMBASE), compreendendo publicações de 1966 até 2011. Resultados e discussão: Foram encontrados nove estudos clínicos considerados pertinentes ao tema. Desses trabalhos, quatro identificaram HS em indivíduos com lesão do LCA e um apontou correlação entre HS e outros fatores de risco para lesões do LCA. A não identificação de correlação entre essas variáveis nos demais estudos se deve, provavelmente, a diferenças metodológicas nas avaliações. Deve-se ressaltar que a natureza retrospectiva dos estudos encontrados não permite o estabelecimento de causa e consequência nesse contexto. São necessários estudos prospectivos, com mais uniformidade metodológica, para o definitivo estabelecimento da HS como efetivo fator de risco para as lesões do LCA.

Palavras-chave: Pronação. Articulação talocalcânea. Ligamento cruzado anterior. Articulação do joelho.

\section{Abstract}

Introduction: Anterior cruciate ligament (ACL) rupture is a severe knee injury, leading to functional instability and degenerative joint disease. Risk factors proximal to the knee joint have been highly emphasized in the last decade, but less attention has been focused on risk factors located distal to the knee. Subtalar hyperpronation (SH) has been suggested by some authors as a possible risk factor for ACL injuries, but the evidences regarding this matter are still scarce and inconclusive. Objective: The purpose of this study was to carry out a review of literature studies that have performed assessments of the subtalar joint alignment associated to ACL injuries or associated to other known risk factors for this injury. Materials and methods: A search in electronic databases (PubMed, MEDLINE, COCHRANE, Web of Science, PEDro, SciELO, LILACS and EMBASE) was performed from 1966 to 2011. Results and discussion: Nine clinical studies were found to be pertinent to this matter. Among these, four studies have identified SH in subjects with ACL injury and one study has found a correlation between SH and other risk factors for ACL injury. The inexistence of correlation between these variables in the other studies is probably due to methodological differences in the assessments. It should be noted that the retrospective nature of the studies found does not allow the establishment of cause and consequence in this context. Prospective studies, with more methodological uniformity, are necessary for the definitive establishment of SH as an effective risk factor for ACL injuries.

Keywords: Pronation. Talocalcaneal joint. Anterior cruciate ligament. Knee joint.

\section{Introdução}

A ruptura do ligamento cruzado anterior (LCA) é uma lesão ligamentar severa, que resulta em instabilidade funcional em curto prazo e distúrbios articulares degenerativos em longo prazo (1). Essa lesão é tradicionalmente descrita como a lesão ligamentar mais comum na articulação do joelho (2), e seu tratamento envolve custo financeiro bastante significativo, considerando-se que é estimado um gasto de aproximadamente 17 mil dólares com intervenção cirúrgica e reabilitação de um paciente com essa lesão. Isso resulta em um gasto superior a 1,7 bilhões de dólares, somente nos Estados Unidos, com o tratamento dessa condição (3). As implicações físicas, mentais, emocionais e econômicas da lesão do LCA têm motivado a realização de análises dos fatores de risco e dos mecanismos envolvidos na lesão, para que estratégias preventivas eficazes possam ser adequadamente estabelecidas $(4,5)$.

Como reportado, entre outros, por Griffin et al. (6), aproximadamente $80 \%$ das lesões do LCA ocorrem sem um trauma direto, o que vem incentivando a comunidade científica a buscar identificar fatores de risco, intrínsecos e extrínsecos ao indivíduo, que possam estar relacionados à lesão. Vários fatores de risco para as lesões do LCA já foram estabelecidos na literatura, incluindo os fatores locais, os proximais e os distais à articulação do joelho $(4,5)$. Fatores locais, como excesso de translação anterior da tíbia em relação ao fêmur, vêm sendo tradicionalmente considerados como aspectos relevantes na gênese da lesão do LCA (7), e, recentemente, alguns fatores de risco mais proximais também têm sido bastante 
enfatizados como fatores predisponentes a essa lesão (8). Alterações cinemáticas no tronco, na pelve e no quadril e alterações de força e recrutamento dos músculos estabilizadores do complexo lombopélvico podem favorecer a ocorrência do chamado valgo dinâmico do joelho, movimento que produz significativo estresse no LCA e o predispõe à lesão (8-10). Outro possível fator de risco, que vem recebendo menos atenção dos estudos da literatura e que se encontra distal à articulação do joelho é uma alteração de alinhamento caracterizada por excesso de pronação da articulação subtalar (hiperpronação subtalar).

Alguns modelos teóricos foram levantados ao longo dos anos, numa tentativa de elucidar os mecanismos pelos quais a hiperpronação da articulação subtalar pode favorecer a ocorrência de lesões no joelho, no quadril e na coluna vertebral (11-13). A hiperpronação subtalar vêm sendo correlacionada a alterações cinemáticas na articulação do joelho (14, 15), com alterações de alinhamento da pelve (16) e com inúmeras lesões, que incluem fraturas por estresse (17), síndrome da dor femoropatelar (18), lesões degenerativas no quadril (19), dentre outras. Além disso, têm sido observadas correlações significativas entre o alinhamento estático da articulação subtalar e as lesões do LCA (20-23), mas ainda há pouco entendimento sobre os mecanismos pelos quais esse mau alinhamento influencia a função neuromuscular e a biomecânica durante atividades esportivas, apresentando-se como fator de risco para essa lesão (24). Melhor entendimento das relações entre as alterações de alinhamento nos pés e as lesões do LCA poderia favorecer a implementação mais efetiva de condutas preventivas abrangentes para a redução nos índices de lesão do LCA.

Considerando-se a origem multifatorial das lesões atraumáticas do LCA (4) e a inerente interrelação existente entre as articulações do membro inferior, torna-se necessária uma abordagem de avaliação abrangente do indivíduo, para a identificação precoce de fatores de risco e a prevenção efetiva da lesão. Apesar das indicações de que existe uma relação entre o excesso de pronação da articulação subtalar e as lesões do LCA (20-23), pouca atenção tem sido dada, especialmente na última década, no sentido de melhor elucidar essa questão, sendo que os resultados dos estudos sobre o tema são contraditórios e pouco conclusivos. Assim, o propósito deste estudo foi realizar uma revisão dos estudos da literatura que tenham buscado identificar alterações de alinhamento da articulação subtalar (hiperpronação subtalar) associadas à lesão do LCA.

\section{Materiais e métodos}

Foi realizada uma busca eletrônica nas bases de dados PubMed, MEDLINE, COCHRANE, Web of Science, PEDro, SciELO, LILACS e EMBASE, abrangendo publicações de 1966 até 2011, no período compreendido entre os dias 25 de abril e 3 de maio de 2011, cruzando-se as seguintes palavras-chave: pronation (pronação), subtalar (subtalar), anterior (anterior), cruciate (cruzado), ligament (ligamento), ACL (LCA) e injury (lesão). Foram incluídos, no presente estudo, os artigos que apresentassem os seguintes critérios: 1) avaliações do alinhamento dos pés (pronação subtalar) associadas a lesões do ligamento cruzado anterior, do joelho de indivíduos de ambos os gêneros, sem distinção de idade; 2) artigos disponíveis na íntegra nos idiomas inglês ou português. Foram excluídos do estudo os trabalhos que analisassem o efeito de palmilhas como condutas de prevenção para a lesão do LCA, uma vez que estávamos interessados em avaliar a relação entre a hiperpronação subtalar e as lesões do LCA, e não o efeito de diferentes condutas preventivas.

Os títulos e os resumos dos trabalhos foram analisados, e os estudos que preenchessem os critérios de inclusão foram obtidos na íntegra. As referências bibliográficas dos estudos também foram analisadas, buscando-se trabalhos que pudessem ser incluídos na revisão. Cada artigo teve seu conteúdo analisado por dois autores separadamente; posteriormente, as informações coletadas por cada autor foram confrontadas, e os dados que divergiram entre os autores foram analisados até que se chegasse a um consenso. 0 conteúdo dos artigos pesquisados deveria apresentar informações relevantes ao tópico explanado e ser passível de comparação com os demais artigos, para que fosse incluído na presente revisão.

\section{Resultados}

Nas bases de dados SciELO, LILACS e PEDro não foram encontrados estudos sobre o presente tema; nas demais bases de dados, nove estudos pertinentes ao tema abordado foram descobertos, todos eles na língua inglesa (20-28). Desses estudos, seis buscaram 
identificar a presença de hiperpronação subtalar em indivíduos com lesão do LCA (20-23, 25, 27), e os três demais avaliaram a relação entre a pronação subtalar e outros fatores de risco para lesões do $\operatorname{LCA}(24,26,28)$.

Com relação às avaliações, oito dos trabalhos avaliaram a pronação subtalar por meio do teste da queda do navicular (20-27), um avaliou a pronação subtalar por análise da distribuição da pressão plantar (28) e nenhum realizou ambas as avaliações do alinhamento dos pés. Todos os trabalhos que avaliaram correlação das lesões de LCA com a hiperpronação subtalar foram análises retrospectivas, ou seja, foi avaliado o alinhamento dos pés de sujeitos que já apresentavam a lesão do LCA.

As principais características dos estudos selecionados estão expostas no Quadro 1.

Quadro 1 - Caracterização dos artigos incluídos na revisão

(Continua)

\begin{tabular}{|c|c|c|c|}
\hline Autores & Caracterização da amostra & Variáveis avaliadas & Resultados \\
\hline $\begin{array}{l}\text { Beckett et al. } \\
(20)\end{array}$ & $\begin{array}{l}\text { Grupo com Lesão do LCA } \\
\text { (GL): } 50 \text { indivíduos ( } 39 \\
\text { homens e } 11 \text { mulheres). } \\
\text { Grupo Controle (GC): } 50 \\
\text { indivíduos sem histórico } \\
\text { de lesão ( } 39 \text { homens e } 11 \\
\text { mulheres). }\end{array}$ & $\begin{array}{l}\text { Foi avaliada a queda do navicular na } \\
\text { transição da posição sentado para a } \\
\text { posição em pé. }\end{array}$ & $\begin{array}{l}0 \text { GL apresentou pronação subtalar } \\
\text { significativamente maior em ambos os } \\
\text { membros inferiores, quando comparado } \\
\text { ao GC. Os valores de queda do navicular } \\
\text { do GL foram, em média, de } 12,85 \mathrm{~mm} \text { e } \\
\text { os do GC foram, em média, de } 6,9 \mathrm{~mm} \text {. }\end{array}$ \\
\hline $\begin{array}{l}\text { Woodford- } \\
\text { Rogers et al. } \\
(21)\end{array}$ & $\begin{array}{l}\text { Grupo com Lesão do LCA } \\
\text { (GL): } 22 \text { indivíduos (14 } \\
\text { homens e } 8 \text { mulheres). } \\
\text { Grupo Controle (GC): } 22 \\
\text { indivíduos sem histórico } \\
\text { de lesão (14 homens e } 8 \\
\text { mulheres). }\end{array}$ & $\begin{array}{l}\text { Foi avaliada a queda do navicular na } \\
\text { transição da posição sentado para } \\
\text { em pé. Além disso, avaliou-se } 0 \\
\text { ângulo de alinhamento do calcâneo } \\
\text { e a translação anterior da tíbia em } \\
\text { relação ao fêmur, com artrômetro } \\
\text { KT-1000. }\end{array}$ & $\begin{array}{l}0 \text { GL apresentou maior queda do } \\
\text { navicular do que } 0 \mathrm{GC} \text { ( } 8,4 \mathrm{~mm} \text { e } 5,9 \\
\mathrm{~mm} \text {, respectivamente). Além disso, } \\
\text { os valores de queda do navicular e de } \\
\text { translação anterior da tíbia na análise } \\
\text { discriminativa foram capazes de predizer } \\
\text { a classificação dos indivíduos nos } \\
\text { respectivos grupos, com precisão em } \\
70,5 \% \text { dos casos. }\end{array}$ \\
\hline $\begin{array}{l}\text { Loudon et al. } \\
\text { (22) }\end{array}$ & $\begin{array}{l}\text { Grupo com Lesão do LCA } \\
\text { (GL): } 20 \text { indivíduos do sexo } \\
\text { feminino. } \\
\text { Grupo Controle (GC): } 20 \\
\text { indivíduos do sexo feminino } \\
\text { sem histórico de lesão. }\end{array}$ & $\begin{array}{l}\text { Foi avaliada a queda do navicular } \\
\text { na transição da posição sentado } \\
\text { para em pé. Além disso, avaliou-se } \\
0 \text { alinhamento pélvico, o ângulo de } \\
\text { anteversão femoral, a flexibilidade } \\
\text { dos isquiotibiais, a presença de } \\
\text { hiperextensão tibiofemoral, o ângulo } \\
\text { Q e a posição neutra da articulação } \\
\text { subtalar. }\end{array}$ & $\begin{array}{l}\text { Somente a queda do navicular } \\
\text { e a presença de hiperextensão } \\
\text { tibiofemoral foram capazes de predizer } \\
\text { a classificação dos indivíduos, nos } \\
\text { respectivos grupos, com precisão na } \\
\text { análise de regressão linear múltipla. Os } \\
\text { valores exatos de queda do navicular não } \\
\text { foram reportados, mas } 15 \text { indivíduos do } \\
\text { GL apresentaram valores maiores do que } \\
9 \mathrm{~mm} \text {. }\end{array}$ \\
\hline Smith et al. (25) & $\begin{array}{l}\text { Grupo com Lesão do LCA } \\
\text { (GL): } 14 \text { indivíduos ( } 7 \text { homens } \\
\text { e } 7 \text { mulheres). } \\
\text { Grupo Controle (GC): } 14 \\
\text { indivíduos sem histórico } \\
\text { de lesão ( } 7 \text { homens e } 7 \\
\text { mulheres). }\end{array}$ & $\begin{array}{l}\text { Foi avaliada a queda do navicular na } \\
\text { transição da posição sentado para } \\
\text { em pé. Além disso, avaliou-se o } \\
\text { ângulo de alinhamento do calcâneo } \\
\text { em apoio bipodal. }\end{array}$ & $\begin{array}{l}\text { Não houve diferença entre os grupos } \\
\text { quanto às variáveis avaliadas. Os valores } \\
\text { de queda do navicular do GL foram, em } \\
\text { média, de } 6,33 \mathrm{~mm} \text { e os do GC foram, } \\
\text { em média, de } 6,21 \mathrm{~mm} \text {. }\end{array}$ \\
\hline $\begin{array}{l}\text { Allen e Glasoe } \\
\text { (23) }\end{array}$ & $\begin{array}{l}\text { Grupo com Lesão do LCA } \\
\text { (GL): } 18 \text { indivíduos (12 } \\
\text { homens e } 6 \text { mulheres). } \\
\text { Grupo Controle (GC): } 18 \\
\text { indivíduos sem histórico } \\
\text { de lesão (12 homens e } 6 \\
\text { mulheres). }\end{array}$ & $\begin{array}{l}\text { Avaliação da queda do navicular em } \\
\text { apoio bipodal. A queda do navicular } \\
\text { foi estabelecida como a diferença } \\
\text { entre a altura do navicular com a } \\
\text { articulação subtalar em posição } \\
\text { neutra e a altura do navicular com os } \\
\text { pés relaxados. Utilizou-se o sistema } \\
\text { Metrecom para a avaliação da altura } \\
\text { do navicular. }\end{array}$ & $\begin{array}{l}0 \text { GL apresentou maior pronação subtalar } \\
\text { em ambos os membros inferiores, } \\
\text { quando comparado ao GC. Os valores } \\
\text { de queda do navicular do GL foram, em } \\
\text { média, de } 10,5 \mathrm{~mm} \text { e os do GC foram, } \\
\text { em média, de } 8,1 \mathrm{~mm} \text {. }\end{array}$ \\
\hline
\end{tabular}


Quadro 1 - Caracterização dos artigos incluídos na revisão

\begin{tabular}{|c|c|c|c|}
\hline Autores & Caracterização da amostra & Variáveis avaliadas & Resultados \\
\hline $\begin{array}{l}\text { Trimble et al. } \\
\text { (26) }\end{array}$ & $\begin{array}{l}43 \text { Indivíduos (16 homens e } \\
27 \text { mulheres) sem histórico } \\
\text { de lesões nos membros } \\
\text { inferiores. } \\
\text { Avaliação de indivíduos } \\
\text { sedentários jovens sadios, } \\
\text { buscando identificar fatores } \\
\text { de risco para lesões do LCA e } \\
\text { correlações entre eles. }\end{array}$ & $\begin{array}{l}\text { Avaliação do alinhamento dos } \\
\text { membros inferiores (queda do } \\
\text { navicular, torção tibial externa } \\
\text { e presença de hiperextensão } \\
\text { tibiofemoral), buscando correlação } \\
\text { dessas variáveis com a translação } \\
\text { anterior da tíbia em relação ao fêmur } \\
\text { (mensurada com o artrômetro KT- } \\
\text { 1000). }\end{array}$ & $\begin{array}{l}\text { A queda do navicular foi a única das } \\
\text { variáveis analisadas que apresentou } \\
\text { correlação significativa com a translação } \\
\text { anterior da tibia. Essa medida de } \\
\text { pronação subtalar foi capaz de explicar } \\
26 \% \text { da variabilidade da translação } \\
\text { anterior da tíbia nas mulheres, na análise } \\
\text { de regressão múltipla. }\end{array}$ \\
\hline $\begin{array}{l}\text { Hargrave et al. } \\
(24)\end{array}$ & $\begin{array}{l}\text { Grupo com Maior Pronação: } \\
16 \text { indivíduos com queda do } \\
\text { navicular (QN) > } 10 \mathrm{~mm} . \\
\text { Grupo com Maior Supinação: } \\
16 \text { indivíduos com QN }<5 \\
\text { mm. } \\
\text { Grupo com Alinhamento } \\
\text { Neutro dos Pés: } 16 \text { indivíduos } \\
\text { com } 5 \mathrm{~mm} \leq \text { QN } \leq 10 \mathrm{~mm} \text {. }\end{array}$ & $\begin{array}{l}\text { Avaliação da força de reação do solo } \\
\text { e da excursão de flexão do joelho } \\
\text { na aterrissagem de salto unipodal, } \\
\text { nos indivíduos com diferentes } \\
\text { alinhamentos dos pés, porém sem } \\
\text { histórico de lesões nos membros } \\
\text { inferiores. }\end{array}$ & $\begin{array}{l}\text { Não se observou diferença entre os três } \\
\text { grupos quanto às variáveis avaliadas. }\end{array}$ \\
\hline $\begin{array}{l}\text { Jenkins et al. } \\
\text { (27) }\end{array}$ & $\begin{array}{l}\text { Grupo com Lesão do LCA } \\
\text { (GL): } 16 \text { indivíduos ( } 2 \text { homens } \\
\text { e } 14 \text { mulheres). } \\
\text { Grupo Controle (GC): } 89 \\
\text { indivíduos sem histórico } \\
\text { de lesão ( } 50 \text { homens e } 39 \\
\text { mulheres). } \\
\text { Os voluntários eram jogadores } \\
\text { de futebol ou basquetebol. }\end{array}$ & $\begin{array}{l}\text { Foi avaliada a queda do navicular } \\
\text { na transiçãa da posiçãao sentado } \\
\text { para a posição em pé. Foi avaliado, } \\
\text { também, o ângulo da articulação } \\
\text { subtalar em posição neutra. }\end{array}$ & $\begin{array}{l}\text { Não houve diferença entre os grupos } \\
\text { quanto às variáveis avaliadas. } 0 \mathrm{GL} \\
\text { apresentou, em média, } 10,6 \mathrm{~mm} \text { de } \\
\text { queda do navicular, ao passo que o GC } \\
\text { apresentou, em média, } 10,0 \mathrm{~mm} \text { ( } \mathrm{p}= \\
0,88 \text { ). }\end{array}$ \\
\hline $\begin{array}{l}\text { Mitchell et al. } \\
\text { (28) }\end{array}$ & $\begin{array}{l}\text { Grupo com predominante } \\
\text { distribuição de pressão } \\
\text { plantar medial: } 34 \text { membros } \\
\text { inferiores. } \\
\text { Grupo com predominante } \\
\text { distribuição de pressão plantar } \\
\text { lateral: } 32 \text { membros inferiores. } \\
\text { As voluntárias eram mulheres } \\
\text { jogadoras de futebol, e a } \\
\text { divisão dos grupos foi feita } \\
\text { por uma avaliação prévia da } \\
\text { distribuição da pressão plantar } \\
\text { na marcha. }\end{array}$ & $\begin{array}{l}\text { Avaliação do tornozelo e do joelho } \\
\text { quanto à cinemática e à cinética } \\
\text { (inversão/eversão e dorsiflexão/ } \\
\text { flexão plantar do tornozelo, flexão/ } \\
\text { extensão e adução/abdução do } \\
\text { joelho), durante uma tarefa de salto } \\
\text { vertical máximo (drop vertical jump). }\end{array}$ & $\begin{array}{l}\text { Não houve diferença entre os grupos, em } \\
\text { nenhuma das variáveis avaliadas, tanto } \\
\text { para a articulação do tornozelo quanto } \\
\text { para a articulação do joelho. }\end{array}$ \\
\hline
\end{tabular}

Fonte: Dados da pesquisa.

\section{Discussão}

A alta incidência, a severidade, o alto custo e a potencial incapacidade em longo prazo associados à lesão do LCA fazem com que a prevenção dessa condição tenha prioridade na comunidade científica (29). A adequada identificação de fatores de risco para essa lesão permite a implementação de condutas preventivas, visando modificar esses fatores, minimizando o índice dessa importante lesão, especialmente nas populações de risco. Na atual revisão, foram encontradas evidências que suportam a hiperpronação subtalar, mensurada por meio do teste da queda do navicular, como potencial fator de risco para as lesões do LCA $(20-23,26)$.

Tiberio (11) foi um dos primeiros autores a sugerir que a hiperpronação subtalar pode influenciar de maneira relevante a gênese e/ou a perpetuação de lesões da articulação do joelho. Sabe-se que a pronação subtalar em cadeia fechada envolve os 
movimentos de eversão do calcâneo, flexão plantar e adução (rotação medial) do tálus (30), e, em função da íntima relação do tálus com a pinça maleolar, os movimentos do tálus resultam em movimentos de rotação medial da tíbia (31). Na fase de apoio médio da marcha, para que o joelho entre em extensão, é necessário que ocorra o mecanismo de aparafusamento tibiofemoral, no qual o fêmur roda para permanecer em rotação medial em relação à tíbia (32). Se ocorrer hiperpronação na articulação subtalar e, consequentemente, um movimento associado de excesso de rotação medial da tíbia, é necessária uma grande quantidade de rotação medial do fêmur, para que a extensão do joelho possa ocorrer (11). Esse movimento rotatório excessivo do membro inferior pode favorecer a ocorrência de inúmeras lesões, como as degenerativas nas articulações do quadril (19) e femoropatelar $(11,12,18)$. Apesar de esse mecanismo ser relativamente bem-estabelecido, a relação exata entre a hiperpronação subtalar e as lesões do LCA ainda não está bem elucidada.

Beckett et al. (20) foram os primeiros autores a identificar excesso de pronação da articulação subtalar em ambos os membros inferiores de 50 indivíduos com ruptura unilateral do LCA, quando comparados a um grupo controle de 50 indivíduos sadios, sem histórico de lesão nos membros inferiores. Os indivíduos foram avaliados quanto à pronação subtalar com o teste de queda do navicular (avaliado como sendo a diferença entre a altura do tubérculo do navicular na posição sentada, estando a articulação subtalar em posição neutra e a altura do tubérculo do navicular na posição ortostática com o pé relaxado, como descrito por Brody (33). No grupo de indivíduos com lesão do LCA, os valores médios de queda do navicular foram de 13,0 $\mathrm{mm}$ no membro inferior direito e de $12,7 \mathrm{~mm}$ no membro inferior esquerdo, ao passo que o grupo controle apresentou, em média, 6,9 mm de queda do navicular, em ambos os membros inferiores (20). Allen e Glasoe (23) também evidenciaram maior queda do navicular bilateralmente, em indivíduos com lesão unilateral do LCA, quando comparados com indivíduos sem histórico de lesão nos membros inferiores. Os autores apresentaram valores de queda do navicular bastante similares aos de Beckett et al. (20), utilizando, porém, avaliações com o Metrecom System, uma ferramenta de análise eletromecânica tridimensional, considerada mais precisa para a avaliação da queda do navicular (23).
Em concordância com esses resultados, WoodfordRogers et al. (21) também evidenciaram maior pronação da articulação subtalar em indivíduos com histórico de lesão do LCA, quando comparados a indivíduos sem lesões nos membros inferiores. Os autores optaram por realizar as avaliações no membro contralateral à lesão, para que possíveis alterações secundárias à lesão não interferissem nos resultados. Foram avaliados, ainda, o ângulo de alinhamento do calcâneo e a translação anterior da tíbia em relação ao fêmur, com um artrômetro KT-1000. Os valores de queda do navicular foram de $8,4 \mathrm{~mm}$ e $5,9 \mathrm{~mm}$, nos grupos com lesão e controle, respectivamente $(\mathrm{p}<$ $0,05)$. Além disso, na análise discriminativa realizada no estudo, os valores de queda do navicular e de translação anterior da tíbia eram capazes de predizer a classificação dos indivíduos nos respectivos grupos, com precisão em 70,5\% dos casos (21).

Loudon et al. (22) avaliaram o alinhamento pélvico, o ângulo de torção femoral, o alinhamento estático do joelho nos planos sagital e frontal, a flexibilidade dos isquiotibiais e a queda do navicular, em 20 indivíduos com histórico de lesão unilateral do LCA e em 20 indivíduos sem histórico de lesão. Na análise de regressão linear múltipla realizada no estudo, somente a queda do navicular e a presença de hiperextensão tibiofemoral foram capazes de predizer a classificação dos indivíduos nos respectivos grupos com precisão (22). Os autores ressaltaram a importância de se considerar esses aspectos, quando combinados, como importantes fatores de risco para a lesão do LCA e enfatizaram a importância de condutas para prevenção de hiperextensões do joelho e para correção da hiperpronação subtalar (22).

Buscando identificar correlações entre diferentes variáveis reconhecidas como fatores de risco para lesões do LCA, Trimble et al. (26) avaliaram 43 indivíduos sem histórico de lesão nos membros inferiores quanto à queda do navicular, quanto ao ângulo de torção tibial, quanto à translação anterior da tíbia em relação ao fêmur e quanto à presença de hiperextensão tibiofemoral. Os autores identificaram que houve correlação significativa entre a hiperpronação subtalar (maior queda do navicular) e uma maior translação anterior da tíbia em relação ao fêmur, nos indivíduos avaliados, de modo que a queda do navicular foi capaz de explicar $26 \%$ da variabilidade da translação anterior da tíbia, em análise de regressão linear múltipla (26). Esses achados de correlação entre a hiperpronação subtalar e o excesso de translação anterior da 
tíbia, um reconhecido fator de risco para as lesões do LCA (7), parecem indicar que o excesso de pronação da articulação subtalar pode favorecer, mesmo que indiretamente, a ocorrência da lesão.

Discordando dos resultados da literatura prévia, Smith et al. (25) e Jenkins et al. (27) não observaram diferença nos valores de queda do navicular ao compararem indivíduos com lesão do LCA e indivíduos sadios de um grupo controle. Nesses estudos, também não houve, entre os grupos, diferenças entre o ângulo do calcâneo e o ângulo da articulação subtalar em posição neutra, apesar de ter sido utilizada uma metodologia similar aos estudos anteriores $(25,27)$. Esses resultados contraditórios indicam que não existe um consenso na literatura sobre o estabelecimento da hiperpronação subtalar como fator de risco efetivo para as lesões do LCA.

Os trabalhos de Hargrave et al. (24) e Mitchell et al. (28) buscaram avaliar a relação entre a hiperpronação subtalar e outros fatores de risco para lesões do LCA. Hargrave et al. (24) avaliaram as forças de reação do solo e a cinemática do joelho no plano sagital durante a aterrissagem de salto unipodal, comparando indivíduos com maior pronação subtalar, indivíduos com alinhamento subtalar neutro e indivíduos com maior supinação subtalar. Não se observou diferença entre os grupos quanto às variáveis avaliadas. Mitchell et al. (28) avaliaram a cinética e a cinemática do joelho e do tornozelo nos planos sagital e coronal, em uma tarefa de aterrissagem de salto bipodal em indivíduos com maior pronação subtalar, comparando-os com indivíduos com maior supinação subtalar. Também não foi observada diferença entre os grupos nas variáveis avaliadas. Esses resultados poderiam indicar que diferenças no alinhamento da articulação subtalar pouco influenciam a capacidade de dissipação de força e a cinemática do membro inferior. Deve-se ressaltar, porém, que esses resultados provavelmente se devem à tarefa avaliada nesses estudos. Ambos os trabalhos avaliaram os voluntários em uma tarefa de aterrissagem de salto, atividade em que o pé apresenta uma sequência de contato com o solo que progride do antepé para o retropé, ao contrário do que ocorre na marcha e na corrida, nas quais a sequência predominante de movimento progride do retropé para o antepé $(11,34)$. É possível que, em uma atividade de sequência de antepé para retropé, o comportamento da articulação subtalar seja diferente do observado em tarefas com sequência que segue do retropé para o antepé (24), de maneira que a articulação subtalar teria menor relevância, em dissipação de força, em tarefas como a aterrissagem de salto.

Outra explicação para não terem sido observadas diferenças entre os grupos nos estudos de Hargrave et al. (24) e Mitchell et al. (28) é o fato de que a pronação subtalar está associada, principalmente, a movimentos do membro inferior no plano transverso, resultando em rotações mediais da tíbia e do fêmur, em atividades em cadeia cinética fechada $(11,35)$. A não avaliação de movimentos no plano transverso nesses estudos pode justificar a ausência de diferenças significativas entre os grupos, quanto às variáveis analisadas.

Os mecanismos pelos quais a hiperpronação da articulação subtalar poderiam contribuir para as lesões sem contato (atraumáticas) do LCA ainda não estão bem esclarecidos. Considerando-se que foi demonstrado que o LCA se tensiona durante a rotação medial da tíbia (36), e que a hiperpronação subtalar está associada a maior rotação medial da tíbia em atividades em cadeia cinética fechada $(11,14,16)$, é possível que essa rotação medial excessiva esteja relacionada a um pré-tensionamento do LCA, mais sujeito a rupturas ao ser submetido a estresse adicional $(20,22,37)$. Outro aspecto a se considerar é o fato de que os mecanismos de lesão sem contato do LCA vêm sendo descritos como os que envolvem movimentos de excessivo valgo dinâmico do joelho, nos quais o fêmur se encontra em adução e rotação medial, a tíbia encontra-se em abdução e rotação lateral e o pé encontra-se fixo no solo em pronação (4, 38), de maneira que o excesso de pronação poderia favorecer tal mecanismo.

Por fim, a hiperpronação subtalar pode, também, contribuir para lesões sem contato do LCA, por um mecanismo indireto. De acordo com hipóteses teóricas e com algumas evidências recentes da literatura, a hiperpronação subtalar apresenta repercussões significativas em toda a cadeia cinética, ao produzir movimentos excessivos de rotação medial em todo o membro inferior $(11,13,16)$. Foi demonstrado, recentemente, que de fato existe correlação significativa entre os movimentos de pronação/supinação da articulação subtalar e os movimentos de rotação medial/rotação lateral do quadril durante a marcha (35). É provável que a pronação excessiva da articulação subtalar gere alterações adaptativas de rotação medial excessiva do fêmur, em cada ciclo da marcha, e é possível que tal evento atue como estímulo para 
que os músculos rotadores laterais do quadril tenham sua força excêntrica reduzida. A redução da força excêntrica dos músculos rotadores laterais do quadril tem sido apontada como causadora de excesso de adução e rotação medial do fêmur em atividades em cadeia cinética fechada (8), com movimentos que compõem o valgo dinâmico do joelho, o que resulta em significativo estresse no LCA, predispondo-o a lesões $(8,9,10)$.

A controvérsia, na literatura, sobre a relação entre a hiperpronação subtalar e as lesões do LCA, com grande variabilidade nos resultados dos diferentes estudos, deve-se a algumas diferenças metodológicas, principalmente quanto às amostras de cada estudo (27). Os estudos de Beckett et al. (20), WoodfordRogers et al. (21), Loudon et al. (22) e Allen e Glasoe (23) encontraram correlação entre elevados valores de queda do navicular com lesões do LCA, ao passo que os trabalhos de Smith et al. (25) e de Jenkins et al. (27) não observaram tal correlação. Os estudos de Smith et al. (25) e de Jenkins et al. (27) utilizaram amostras com menor número de homens com lesão do LCA, o que implicou valores de queda do navicular menores do que os observados nos demais estudos. Acredita-se que a maior estatura dos homens esteja relacionada a um arco plantar mais elevado e que esse fator, associado à maior massa corporal dos homens, possa resultar em maior excursão de queda do navicular em situações de suporte do peso corporal $(21,25)$.

Apesar de algumas evidências suportarem a hiperpronação subtalar como importante fator de risco para lesões do LCA, deve-se ter em mente que essa lesão apresenta origem multifatorial, envolvendo aspectos locais, proximais e distais à articulação do joelho, e as medidas do alinhamento dos pés poderiam ser pouco abrangentes para explicar a etiologia da lesão (27). Existe ainda muita controvérsia na literatura atual a respeito dos mecanismos biomecânicos relacionados às lesões sem contato do LCA (39). Tem-se estabelecido que um importante mecanismo de lesão é o colapso em valgo do joelho, em situações de mudança de direção ou aterrissagem de salto, quando o joelho se encontra na condição denominada "posição sem retorno", caracterizada pelo excesso de adução e rotação medial do fêmur associado à rotação externa da tíbia, estando o pé fixo no solo, em hiperpronação subtalar (40), principalmente quando há, também, a inclinação do tronco para o lado do membro de apoio (38). Embora os fatores proximais possam apresentar relevância significativa nesse mecanismo, a hiperpronação subtalar não deve ser negligenciada como um dos componentes desse movimento extremo, sendo necessária sua avaliação em programas de prevenção de lesões no joelho.

\section{Conclusão}

Em conclusão, existem evidências na literatura que identificam correlação da hiperpronação subtalar com as lesões do LCA (20-23), porém tais indícios ainda são escassos e pouco conclusivos. Além disso, a natureza retrospectiva desses estudos não permite o estabelecimento de causa e consequência, ou seja, não se sabe ao certo se a hiperpronação subtalar foi verificada nos indivíduos como consequência da lesão, ou se foi um fator de risco para ela. Dessa forma, são necessários estudos prospectivos, com mais uniformidade metodológica, para o definitivo estabelecimento da hiperpronação subtalar como efetivo fator de risco para as lesões do LCA.

\section{Referências}

1. Hewett TE, Lynch TR, Myer GD, Ford KR, Gwin RC, Heidt RS Jr. Multiple risk factors related to familial predisposition to anterior cruciate ligament injury: fraternal twin sisters with anterior cruciate ligament ruptures. Br J Sports Med. 2010;44(12):848-55. doi:10.1136/ bjsm.2008.055798.

2. Johnson RJ. The anterior cruciate ligament problem. Clin Orthop. 1982;(172):14-8. PMid:6805994.

3. Hewett TE, Lindenfeld TN, Riccobene JV, Noyes FR. The effect of neuromuscular training on the incidence of knee injury in female athletes: a prospective study. Am J Sports Med. 1999;27(6):699-706. PMid:10569353.

4. Hewett TE, Myer GD, Ford KR. Anterior cruciate ligament injuries in female athletes. Part 1: mechanisms and risk factors. Am J Sports Med. 2006;34(2):299311. doi: $10.1177 / 0363546505284183$.

5. Alentorn-Geli E, Myer GD, Silvers HJ, Samitier G, Romero D, Lázaro-Haro C, etal. Prevention of non-contact anterior cruciate ligament injuries in soccer players. Part 1: mechanisms of injury and underlying risk factors. Knee Surg Sports Traumatol Arthrosc. 2009;17(7):70529. doi:10.1007/s00167-009-0813-1. 
6. Griffin LY, Agel J, Albohm MJ, Arendt EA, Dick RW, Garrett WE, et al. Non-contact anterior cruciate ligament injuries: risk factors and prevention strategies. J Am Acad Orthop Surg. 2000;8(3):141-50. PMid:10874221.

7. Uhorchak JM, Scoville CR, Williams GN, Arciero RA, St Pierre P, Taylor DC. Risk factors associated with non-contact injury of the anterior cruciate ligament: a prospective four-year evaluation of 859 West Point cadets. Am J Sports Med. 2003;31(6):831-42. PMid:14623646.

8. Powers CM. The influence of abnormal hip mechanics on knee injury: a biomechanical perspective. J Orthop Sports Phys Ther. 2010;40(2):42-51. PMid:20118526.

9. Fung DT, Zhang LQ. Modeling of ACL impingement against the intercondylar notch. Clin Biomech. 2003;18(10):933-41. doi:10.1016/S02680033(03)00174-8.

10. Hewett TE, Myer GD, Ford KR, Heidt RS, Colosimo AJ, McLean SG, et al. Biomechanical measures of neuromuscular control and valgus loading of the knee predict anterior cruciate ligament injury risk in female athletes: a prospective study. Am J Sports Med. 2005;33(4):492-501. doi:10.1177/0363546504269591.

11. Tiberio D. The effect of excessive subtalar joint pronation on patellofemoral joint mechanics: a theoretical model. J Orthop Sports Phys Ther. 1987;9(4):160-5. PMid:18797010.

12. Powers CM. The influence of altered lower-extremity kinematics on patellofemoral joint dysfunction: a theoretical perspective. J Orthop Sports Phys Ther. 2003;33:639-46. PMid:14669959.

13. Fonseca ST, Ocarino JM, Silva PLP, Aquino CF. Integration of stress and their relationship to the kinetic chain. In: Magee DJ, Zachazewski JE, Quillen WS, editor. Scientific foundations and principles of practice in musculoskeletal rehabilitation. St Louis: Saunders Elsevier; 2007. p. 476-86.

14. Coplan JA. Rotational motion of the knee: a comparison of normal and pronating subjects. J Orthop Sports Phys Ther. 1989;11:366-9.

15. McClay I, Manal K. A comparison of three-dimensional lower extremity kinematics during running between excessive pronators and normals. Clin Biomech. 1998;13(3):195-203. doi:10.1016/ S0268-0033(97)00029-6.
16. Khamis S, Yizhar Z. Effect of feet hyperpronation on pelvic alignment in a standing position. Gait Posture. 2007;25(1):127-34. doi:10.1016/j. gaitpost.2006.02.005.

17. Hintermann B, Nigg BM. Pronation in runners: implications for injuries. Sports Med. 1998;26(3):169-76. doi:10.2165/00007256-199826030-00003.

18. Boling MC, Padua DA, Marshall SW, Guskiewicz K, Pyne $S$, Beutler A. A prospective investigation of biomechanical risk factors for patellofemoral pain syndrome: the joint undertaking to monitor and prevent ACL injury cohort. Am J Sports Med. 2009;37(11):2108-16. doi:10.1177/0363546509337934.

19. Gross KD, Niu J, Zhang YQ, Felson DT, McLennan C, Hannan MT, et al. Varus foot alignment and hip conditions in older adults. Arthr Rheum. 2007;56(9):29938. doi:10.1002/art.22850.

20. Beckett ME, Massie DL, Bowers KD, Stoll DA. Incidence of hyperpronation in the ACL injured knee: a clinical perspective. J Athl Train. 1992;27(1):58-62. PMid:16558134.

21. Woodford-Rogers B, Cyphert L, Denegar CR. Risk factors for anterior cruciate ligament injury in high school and college athletes. J Athl Train. 1994; 29(4):343-6. PMid:16558298.

22. Loudon JK, Jenkins W, Loudon KL. The relationship between static posture and ACL injury in female athletes. J Orthop Sports Phys Ther. 1996;24(2):91-7. PMid:8832472.

23. Allen MK, Glasoe WM. Metrecom measurement of navicular drop in subjects with anterior cruciate ligament injury. J Athl Train. 2000;35(4):403-6. PMid:16558652.

24. Hargrave MD, Carcia CR, Gansneder BM, Shultz SJ. Subtalar pronation does not influence impact forces or rate of loading during a single-leg landing. J Athl Train. 2003;38(1):18-23. PMid:12937467.

25. Smith J, Szczerba JE, Arnold BL, Perrin DH, Martin DE. Role of hyperpronation as a possible risk factor for anterior cruciate ligament injuries. J Athl Train. 1997;32:25-8. PMid:16558428.

26. Trimble MH, Bishop MD, Buckley BD, Fields LC, Rozea GD. The relationship between clinical measurements of lower extremity posture and tibial translation. Clin Biomech. 2002;17:286-90. doi:10.1016/ S0268-0033(02)00010-4. 
27. Jenkins WL, Killian CB, Williams DS 3rd, Loudon J, Raedeke SG. Anterior cruciate ligament injury in female and male athletes: the relationship between foot structure and injury. J Am Podiatr Med Assoc. 2007;97(5):371-6. PMid:17901341.

28. Mitchell LC, Ford KR, Minning S, Myer GD, Mangine RE, Hewett TE. Medial foot loading on ankle and knee biomechanics. N Am J Sports Phys Ther. 2008;3(3):13340. PMid:20379384.

29. Bonci CM. Assessment and evaluation of predisposing factors to anterior cruciate ligament injury. J Athl Train. 1999;34(2):155-64. PMid:16558559.

30. Johanson MA, Donatelli R, Wooden MJ, Andrew PD, Cummings GS. Effects of three different posting methods on controlling abnormal subtalar pronation. Phys Ther. 1994;74(2):149-58. PMid:8290620.

31. Rockar PA Jr. The subtalar joint: anatomy and joint motion. J Orthop Sports Phys Ther. 1995;21(6):36172. PMid:7655480.

32. Moglo KE, Shirazi-Adl A. Cruciate coupling and screw-home mechanism in passive knee joint during extension-flexion. J Biomech. 2005;38(5):1075-83. doi:10.1016/j.jbiomech.2004.05.033.

33. Brody DM. Techniques in the evaluation and treatment of the injured runner. Orthop Clin North Am. 1982;13(3):541-58. PMid:6124928.

34. Purcell S. The causes of hyperpronation of the foot and resultant pathologies in the runner. J Can Athl Ther Assoc. 1986;13:9-12.
35. Souza TR, Pinto RZ, Trede RG, Kirkwood RN, Fonseca ST. Temporal couplings between rearfoot-shank complex and hip joint during walking. Clin Biomech. 2010;25(7):745-8. doi:10.1016/j. clinbiomech.2010.04.012.

36. Markolf KL, Burchfield DM, Shapiro MM, Shepard MF, Finerman GA, Slauterbeck JL. Combined knee loading states that generate high anterior cruciate ligament forces. J Orthop Res. 1995;13(6):930-5. doi:10.1002/ jor.1100130618.

37. Fish DJ. Ligament integrity protects against stress and strain. Biomechanics. 1998;5:49-53.

38. Hewett TE, Torg JS, Boden BP. Video analysis of trunk and knee motion during non-contact anterior cruciate ligament injury in female athletes: lateral trunk and knee abduction motion are combined components of the injury mechanism. Br J Sports Med. 2009;43(6):417-22. doi:10.1136/bjsm.2009.059162.

39. Quatman CE, Hewett TE. The anterior cruciate ligament injury controversy: is 'valgus collapse' a sex-specific mechanism? Br J Sports Med. 2009;43(5):328-35. doi:10.1136/bjsm.2009.059139.

40. Ireland ML. Anterior cruciate ligament injury in female athletes: epidemiology. J Athl Train. 1999;34(2):150-4. PMid:16558558.

Recebido: 09/08/2011

Received: 08/09/2011

Aprovado: 02/02/2012

Approved: 02/02/2012 